\title{
Value of FDG PET/CT in the Management of
}

\section{Mesothelioma}

\author{
Mohamad Faizul Abu Hanifa ${ }^{1}$, Mehdi Taghipour Fard Ardekani ${ }^{2}$ and Rathan Subramaniam ${ }^{2}$ \\ 1. Perdana University Graduate School of Medicine, Kuala Lumpur 50000, Malaysia \\ 2. The Russell H. Morgan Department of Radiology and Radiological Science, Johns Hopkins Medicine, Baltimore 21231, USA
}

\begin{abstract}
The aim of this study is to compare and discuss the efficacy of FDG PET/CT (fludeoxyglucose positron emission tomography/computated tomography) in the diagnosis, staging, prognosis, pre-therapeutic planning, therapeutic assessment and detecting recurrence of mesothelioma. Following method has been used: (1) Search setting on PubMed (by priority): meta-analysis, randomized controlled trial, other study types; (2) The key words: "FDG PET/CT”, “mesothelioma”, “diagnosis”, "staging”, "prognosis", "therapeutic", "assessment” and "recurrences”; (3) Review the literature that discusses the imaging modalities in the management of mesothelioma; (4) Create an evidence table; (5) Compare and discuss the different modalities and their sensitivity and specificity in managing mesothelioma; (6) Construct the passage reviewing the articles chosen. There is evidence that suggests that FDG PET/CT is superior to most other modalities in the diagnosis, staging, prognosis, pre-therapeutic planning, therapeutic assessment and detecting recurrence of mesothelioma. There are some close contenders like PET scan by itself as a modality to detect mesothelioma. However, due to the lack of anatomical presentation, there were many occurrences of misdiagnosis. FDG PET/CT, which superimposes functional imaging over the anatomical mapping, yields a more accurate presentation of mesothelioma. The nature of this hybrid imaging enables diagnosis and management to be carried-out in a minimally invasive manner. PET/CT to a great extent has tranformed the way we diagnose neoplastic occurences heretofore. By amalgamating anatomic localization to functional imaging, this hybrid modality yields valuable information that was remotely attainable before. Although there are a few flaws to PET/CT that have been discussed in some studies, there is an apparent potential to this modality that is waiting to be uncovered. Evidently, PET/CT is highly effective in differentiating benign and malignant pleural lesion. Likewise, it is substantially useful in the management of mesothelioma particularly in the diagnosis, staging, prognosis, treatment, monitoring and detecting recurrences. With PET/CT having such adequacy in managing mesothelioma, it is imperative to acknowledge and further investigate this modality as a central tool in the management of the latter for the exclusive benefit of the patient.
\end{abstract}

Key words: FDG PET/CT, mesothelioma, diagnosis, staging, prognosis, therapeutic, assessment, recurrences.

\section{Introduction}

In the human embryological development, the body cavity (coelom) is lined with cell layers originating from the embryonic mesoderm. These cells are usually simple squamous cells that constitute a protective layer of the internal organs in the body forming the mesothelium [1]. Mesothelioma is a tumor that develops from the mesothelium. Every year, there is an estimated incidence of 2,000 to 2,500 cases of mesothelioma [2]. Between the year 1994 and 2008, a total of 92,253 mesothelioma-related deaths were

Corresponding author: Mohamad Faizul Abu Hanifa, M.D., research fields: radiology and neuroscience. reported to the WHO (World Health Organization) [3]. In the US, nearly 3,000 deaths per year are attributable to mesothelioma, while the number is around 5,000 in Western Europe [4]. The mean age of death due to mesothelioma was reported as 70 years and male-to-female ratio was 3.6:1. The age-adjusted mortality rate was 4.9 per million populations with an increment of $5.37 \%$ per year. This has resulted in a more than two-fold of increase in mesothelioma related death within 14 years of analysis [3]. Upon diagnosis, the median survival remains less than 12 months in most cases [5]. This makes mesothelioma one of the cancers with the worst prognosis. Mesothelioma presents with several symptoms respective to the site of 
the cancer. Pleural mesothelioma for instance, presents with breathlessness (due to pleural effusion) $(60 \%)$, chest pain $(60 \%)$, weight loss and fatigue $(30 \%)$. On the other hand, peritoneal mesothelioma usually presents with abdominal distension or pain. Noticeable signs, just like the symptoms, are also location inclined. The most common findings for pleural mesothelioma include pleural effusion, fixed hemithorax and reduced breath sounds. Ascites, palpable abdominal masses and abdominal tenderness are typical for peritoneal mesothelioma, while superior vena cava obstruction, phrenic nerve and esophageal constriction is associated with pericardial mesothelioma [6]. The most commonly used classification method for mesothelioma would be the TNM (tumor-lymph nodes-metastasis based) staging proposed by the AJCC (American Joint Committee of Cancer). It addresses the features of the main tumor (T), lymph node involvement $(N)$ and spread of the cancer out of the primary site $(M)$. In a study by Rusch and Venkatraman [7], the TNM staging is deduced to be favorable in influencing the outcome and identifying patients whose prognoses are poor.

The association of asbestos and mesothelioma had been long studied and asbestos has been concluded as an indisputable risk factor for mesothelioma [8]. Asbestos has been causally linked with mesothelioma with an etiological fraction of $80 \%$ or more [9]. Nevertheless, mesothelioma has been documented in patients without prior history of exposure to asbestos and there are reports that showed association between some other risk factors, such as irradiation, thorium dioxide exposure, exposures to fibrous forms of erionite and simian virus 40 and mesothelioma but the incidence is rare [10-15].

Since its inception in the early 1990s, PET/CT (positron emission tomography/computated tomography) has been gaining popularity amongst radiologists [16]. In the past decade, FDG (fludeoxyglucose) PET/CT had gained so much attention that researchers are galloping in the race to discover the whole potential of PET/CT. In the heat of all these, this review would serve to discuss the value of FDG PET/CT in the diagnosis, staging, prognosis, treatment and detecting recurrence of mesothelioma.

\section{Conventional Diagnosis and Imaging}

Diagnosis of mesothelioma is often difficult as it shares features with a number of other similar conditions. A typical diagnosis will take path from medical history and physical examination followed by imaging studies, blood tests and finally biopsies. X-ray radiographic finding of pleural effusion or pleural thickening is a feature of mesothelioma [17]. Although $\mathrm{X}$-ray is a cheap and quick method to get a radiographic image, it does not provide a conclusive indication for mesothelioma. It only hints that there is an abnormal density that requires further investigation. Hence it is a good first test to eliminate the possibility of mesothelioma but a poor confirmatory diagnostic test for the aforementioned [18].

In comparison to X-ray, CT (computed tomography) provides a greater detail of bones, soft tissues and blood vessels. Nonetheless, conventional CT has poor sensitivity and specificity in diagnosing pleural abnormality [19]. Truong et al. [20] discussed in a review on the role of $\mathrm{CT}$, MRI (magnetic resonance imaging) and PET/CT in staging evaluation and treatment consideration in the case of MPM (malignant pleural mesothelioma). It was cited that due to their inability to identify microscopic disease, $\mathrm{CT}$ and MRI have poor accuracy in detecting transdiaphragmatic extension of MPM [20]. In fact, a study to detect and distinguish the pleural disease in 34 patients, carried out by Knuuttila et al. [21], had reported that the MRI provided more information than enhanced CT in differentiating malignant from benign pleural disease. In other words, the characteristic findings in mesothelioma of focal thickening, enhancement of interlobar fissures, tumor invasion of the diaphragm, mediastinal soft tissue or chest wall, are better detected through MRI than CT [21]. This limited accuracy in the 
differentiation between benign and malignant pleural disease is the major hiccup in CT scan assessment. MRI, on the other hand, is generally used to image soft tissues in the body. There are some studies that had shown that MRI is useful in assessing mesothelioma. Entwisle [22] reviewed that although MRIs are not routinely used for mesothelioma assessment, it does shine some light when it comes to differentiating between benign and malignant pleural disease. Hence, it further aids in the process of determining the treatment fashion of whether the patient is a good candidate for radical surgery [22]. In another study by Boraschi et al. [23], the sensitivity, specificity and diagnostic accuracy of the MRI in classifying a lesion as indicative of malignancy were $100 \%, 95 \%$ and $97 \%$, respectively, in a total of 30 patients with asbestos-related pleural disease [23]. Still, MRI does not generate image quality sufficient for a definitive diagnosis within a reasonable examination time [24]. A study by Patel et al. [13] discussed that the relatively high cost, long imaging times and various other contraindications, such as for people who have cardiac pacemaker, defibrillator leads, metal implants, or cerebral aneurysm clips, make MRI a second-line modality to specifically detect peritoneal deposit [13]. As such, MRI is not considered a routine assessment for mesothelioma unless invasion of chest wall or diaphragm is queried [25].

Tumor cells are metabolically active. Therefore, a modality that observes the metabolic intake of cells would project tumor cells well. This is the notion behind PET (positron emission tomography). PET scan relies on a positron-emitting radionuclide (tracer) that is transfused into the patient to emit gamma rays that are detected by the scanner. FDG PET is generally good in differentiating benign from malignant pleural mesothelioma, which helps in detecting recurrence and provides prognostic information (staging, survival, mortality, etc.), mentioned a review by Zahid et al. [26]. Wang et al. [27] published a meta-analysis of seven studies with a total of 267 patients concluding that
FDG PET and PET/CT showed a high sensitivity in detecting cancers (thyroid) in patients with indeterminate fine needle aspiration biopsy results. In the analysis, the best cut-off value of standardized uptake value $S U V_{\max }$ for differentiation was 2.05 but with a high sensitivity of $89.8 \%$ and low specificity of $42.0 \%$ [28]. As good as PET scan is in detecting tumor cells through the increased intake (accummulation) of the radionuclide tracer, it lacks the anatomical propensity that helps to build a solid diagnostic assessment for mesothelioma. This drawback is effortlessly alleviated in FDG PET/CT modality. FDG PET/CT combines the functional imaging of FDG PET and anatomical representation of $\mathrm{CT}$ in a single imaging procedure to produce a highly accurate image for improved management of the disease [24].

\subsection{Significance of PET/CT in the Diagnosis of Mesothelioma}

Diagnosing mesothelioma is the first step in management of the disease. As intuitive as it sounds, reaching the correct diagnosis is extremely difficult given the nature of the disease that mimics other cancers [29]. The current protocol for the diagnosis of mesothelioma involves histopathological assessment including immunohistochemistry as the gold standard [30]. However, the preparation for this test requires biopsy to obtain the specimen desired. This carries a risk of the tumor seeding elsewhere or other general risk associated with surgery, such as hemorrhagic shock or even infection. FDG PET/CT combines functional imaging with anatomical representation to produce a model that could be used for the diagnosis. A meta-analysis of 16 studies comprising of 745 patients revealed that PET and PET/CT has superior diagnostic accuracy for pleural lesions compared to CT alone, with good sensitivity (95\%), specificity (82\%) and pooled sensitivity $(A U C=0.95)$. Notwithstanding, a sub-analysis within the same study shows only very small increase in diagnostic accuracy of PET/CT 
compared to PET. Perhaps the low number of studies included in the meta-analysis and false findings could be a reason for the insignificant difference between the two modalities [31]. Another study by Orki et al. [28] testified that PET/CT imaging had 100\% sensitivity, $94.8 \%$ specificity and $97.5 \%$ accuracy in differentiating benign and malignant pleural disease. Of the studied 83 patients, histopathological examinations revealed malignancy in 44 patients and benign tumor in the other 39. PET/CT was positive for all the malignancy cases with no false negatives. However, there were two false positive cases involving patients with tuberculosis [28]. This is due to the active inflammatory reactions occurring in the patients with tuberculosis leading to increased FDG uptake by the affected cells (standardized uptake value $S U V>3.0$ ) that could be mistaken for malignancy in the PET/CT scanning.

\subsection{Value of PET/CT on the Staging of Mesothelioma}

The staging of mesothelioma is perhaps the most discussed portion of the management by both clinicians and researchers. In many ways, it helps to classify patients into groups that represent certain prognoses and treatments. Plathow et al. [32] substantiated that PET/CT was significantly more accurate $(P<0.005)$ compared to CT, PET and MRI in staging pleural mesothelioma. This study included 54 patients with epithelial MPM found PET/CT had an accuracy of $100 \%$ in all tumor stage. In Stages II and III of the disease, the accuracy was significantly better than all other methods [32].

In the management of mesothelioma, it is crucial to assign the patient's condition to the appropriate stage. Only from the correct identification of the stage can the treatment be planned accordingly. It is rather obvious that the conventional imaging modality is not completely reliable in accurately staging the patient. As a matter of fact, CT could correctly identify a mere $25 \%$ of patients of unresectability, in a clinical study to determine if CT or MRI could accurately predict resectability in patients with biopsy-proved MPM. Likewise, only $50 \%$ of patients with unresectable tumors (during thoracotomy) were found to be unresectable by MRI [30, 32].

\subsection{Importance of PET/CT in Pre-therapeutic Planning}

Treatment is the main stake in any disease management. However, deciding an appropriate treatment is often difficult, especially in a disease like mesothelioma where patients usually present with advanced clinical stage and other comorbidities [33]. PET/CT provides a better picture in deciding the therapeutic intervention in mesothelioma. A prospective study by Feigen et al. [34] showed PET/CT scan based treatment planning and outcome assessment is useful in the preparation and monitoring treatment regimens in MPM patients. Another study of 13 patients that retrospectively compared PET/CT and CT in tumor staging and hence, therapeutic planning of MPM patients, found PET/CT to have reduce geographic misses and significantly decrease target volumes which have clinical benefits and tumor control rates. Twelve out of 13 patients with PET/CT based delineation (in comparison with CT based delineation) resulted in a statistically significant decrease in mean GTV (gross tumor volume), CTV (clinical target volume), PTV1 (planning target Volume 1) and PTV 2 (planning target Volume 2). In 4 out of 13 patients, hilar lymph nodes were identified by PET/CT that was not picked-up by CT alone [35].

\subsection{Prospect of PET/CT in Therapeutic Assessment}

One of the most puzzling aspects in the management of any cancer is the assessment of the effectiveness of a therapy. This is crucial, as failure of response to therapy should be detected early for swift change in the management for the disease. There are many studies supporting the notion of PET/CT as the ideal modality to monitor response of the therapy of mesothelioma [36-40]. As matter of fact, Veit-Haibact et al. [41] in a prospective study of 41 patients summarized that the 
response evaluation by $\mathrm{PET} / \mathrm{CT}$ are significantly predictive for survival. There are also additional findings of the specific parameters that contribute more towards survival (CT, RECIST (response evaluation criteria in solid tumors) and PET, TLG/PETvol (tumor lesion glycolysis/tumor volume) more than $\left.S U V_{\max }\right)$ [41]. In agreement with that, another analysis by Niccoli-Asabella et al. [42] concluded that FDG $\mathrm{PET} / \mathrm{CT}$ is useful to monitor the follow-up and assess the metabolic response to chemo and radiotherapy. This study compared PET/CT with standard diagnostic follow-up CECT (contrast-enhanced computed tomography). While the sensitivity was high in both PET/CT (100\%) and CECT (96.2\%), the specificity was higher in PET/CT (100\%) compared to CECT $(20 \%)$ [42].

\subsection{Detecting Recurrence of Mesothelioma Using PET/CT}

Treatment is often taken for granted as the end-point of cancer management. On the contrary, clinicians would agree that recurrence of cancer in previously treated patient is always a concern. There are reports describing the patterns of disease recurrence after treatment and factors that are linked with the recurrences [43, 44]. Hence, it is equally important to accurately diagnose disease recurrence in order to galvanize a suitable response plan. Twenty-five out of 44 patients had PET/CT performed after a multimodal therapy in a retrospective study by Tan et al. [45] and showed that PET/CT precisely diagnosed recurrent disease in eight patients. With a sensitivity of $94 \%$, specificity of $100 \%$, positive predictive value of $100 \%$ and negative predictive value of $88 \%$, it was settled that PET/CT is of valuable importance in diagnosing recurrence after multimodal therapy for MPM. In the same tune, Bille et al. [46] found that there was a statistically significant correlation between the $S U V_{\max }$ and TLG (tumor lesion glycolysis) of recurrent mesothelioma $(P=0.05)$.

\subsection{Value of PET/CT on the Prognosis of Mesothelioma}

Knowing the predicted outcome of a disease is discouraging and often unpleasant for the patients, but for the physicians it gives the advantage of planning for a customized treatment fitting for the patient. This way, no one will be under-treated nor over-treated. Patients simply receive what is sufficient for them to continue surviving against the disease while having to deal less with unnecessary side effects. Mesothelioma in general, presents with a poor prognosis. There are many dynamics involved in predicting the prognosis of the disease. A number of articles have cited $S U V_{\max }$ as an indicator for the prognosis of patients with mesothelioma. $S U V_{\max }$ is used in PET imagings as a tool of semiquantitative analysis. It provides a ratio of a specific tissue tracer (FDG) uptake standardized to the dose of the tracer administered [47]. This is essential to avoid confounding factors of heterogeneity in terms of body weights of patients and hence, establish a standard reference range, modified from text: Clinical PET and PET/CT by Jadvar et al. [48] and Masa-Ah et al. [49].

$S U V[t]=\frac{\text { Concentration }[t](M B q / \mathrm{kg})}{\text { Injected Activity }[t](M B q) \div \text { Body Weight }(\mathrm{kg})}$ where:

Concentration: concentration of the radioactive tracer in tissue at time $t$;

$M B q$ : radiation measurement-megabecquerel;

Injected Activity: concentration of injected radioactive tracer in the body at time $t$;

Body weight: weight of the person in $\mathrm{kg}$.

Many studies have illustrated the prognostic significance of $S U V_{\max }$. For instance, Flores et al. [50] revealed that patients with higher $S U V_{\max }$ values or metastatic disease had a poorer survival [50]. On the contrary, Genestreti et al. [51] did a study to gage whether $S U V_{\max }$ is inversely associated with prognosis of patient with MPM. There were no statistically significant correlation found between $S U V_{\max }$ and survival $[2,51]$. Pulling the pieces of the puzzle together, 
although PET is fairly precise in predicting survival, PET-CT is proven to produce good accuracy and independent prognostic value (by yielding better estimate of locoregional and distant MPM recurrence) that overcomes the limitations of a single modality by itself [52].

\section{Conclusions}

$\mathrm{PET} / \mathrm{CT}$ to a great extent has tranformed the way we diagnose neoplastic occurences heretofore. By amalgamating anatomic localization to functional imaging, this hybrid modality yields valuable information that was remotely attainable before. Although there are a few flaws to PET/CT that have been discussed in some studies, there is an apparent potential to this modality that is waiting to be uncovered. Evidently, PET/CT is highly effective in differentiating benign and malignant pleural lesion. Likewise, it is substantially useful in the management of mesothelioma particularly in the diagnosis, staging, prognosis, treatment, monitoring and detecting recurrences. This paper may serve as a guide to clinicians to consider PET/CT as a routine modality for patients with high-risk, suspected or diagnosed mesothelioma. As mesothelioma is associated with a poor prognosis, clinicians may find the data in this study expedient to add to their clinical judgement in managing patients with the condition. In future, it would be beneficial to conduct and analyze larger scale studies to strengthen the statistical power of the value of PET/CT in the management of mesothelioma. Studies should also include more randomized-controlled trials to enable better evaluation of the modalities that are compared against PET/CT. With PET/CT having such adequacy in managing mesothelioma, it is imperative to acknowledge and further investigate this modality as a central tool in the management of the latter for the exclusive benefit of the patient.

\section{References}

[1] Eroschenko, V. P., and Di Fiore, M. S. H. 2013. Di Fiore's
Atlas of Histology with Functional Correlations. Riverwoods: Wolters Kluwer Health/Lippincott Williams \& Wilkins.

[2] Kruse, M., Sherry, S. J., Paidpally, V., Mercier, G., and Subramaniam, R. M. 2013. "FDG PET/CT in the Management of Primary Pleural Tumors and Pleural Metastases." AJR Am. J. Roentgenol. 201 (2): W215-26.

[3] Delgermaa, V., Takahashi, K., Park, E. K., Le, G. V., Hara, T., and Sorahan, T. 2011. "Global Mesothelioma Deaths Reported to the World Health Organization between 1994 and 2008." Bull. World Health Organ. 89 (10): 716-24.

[4] Ismail-Khan, R., Robinson, L. A., Williams, C. C. Jr., Garrett, C. R., Bepler, G., and Simon, G. R. 2006. "Malignant Pleural Mesothelioma: A Comprehensive Review." Cancer Control 13 (4): 255-63.

[5] Steele, J. P., Klabatsa, A., Fennell, D. A., Palläska, A., Sheaff, M. T., Evans, M. T., et al. 2005. "Prognostic Factors in Mesothelioma." Lung Cancer 49 : S49-52.

[6] Robinson, B. W., Musk, A. W., and Lake, R. A. 2005. “Malignant Mesothelioma.” Lancet 366 (9483): 397-408.

[7] Rusch, V. W., and Venkatraman, E. 1996. "The Importance of Surgical Staging in the Treatment of Malignant Pleural Mesothelioma." J. Thorac. Cardiovasc. Surg. 111 (4): 815-25.

[8] Roggli, V. L., Sharma, A., Butnor, K. J., Sporn, T., and Vollmer, R. T. 2002. "Malignant Mesothelioma and Occupational Exposure to Asbestos: A Clinicopathological Correlation of 1,445 Cases." Ultrastruct. Pathol. 26 (2): 55-65.

[9] McDonald, J. C., and McDonald, A. D. 1996. "The Epidemiology of Mesothelioma in Historical Context." Eur. Respir. J. 9 (9): 1932-42.

[10] Farioli, A., Violante, F. S., Mattioli, S., Curti, S., and Kriebel, D. 2013. "Risk of Mesothelioma Following External Beam Radiotherapy for Prostate Cancer: A Cohort Analysis of SEER Database." Cancer Causes Control 24 (8): $1535-45$.

[11] Gibb, H., Fulcher, K., Nagarajan, S., McCord, S., Fallahian, N. A., Hoffman, H. J., et al. 2013. "Analyses of Radiation and Mesothelioma in the US Transuranium and Uranium Registries." Am. J. Public Health 103 (4): 710-6.

[12] Jin, M. L., Li, X., and Luo, J. 2006. "Relationship between the Malignant Mesothelioma and Simian Virus 40 in China: A Study of 17 Cases." Zhonghua Bing Li Xue Za Zhi 35 (10): 602-5.

[13] Patel, C. M., Sahdev, A., and Reznek, R. H. 2011. “CT, MRI and PET Imaging in Peritoneal Malignancy." Cancer Imaging 11 (1): 123-39.

[14] Rivera, Z., Strianese, O., Bertino, P., Yang, H., Pass, H., and Carbone, M. 2008. "The Relationship between Simian Virus 40 and Mesothelioma." Curr. Opin. Pulm. Med. 14 (4): 316-21. 
[15] Van Gosen, B. S., Blitz, T. A., Plumlee, G. S., Meeker, G. P., and Pierson, M. P. 2013. "Geologic Occurrences of Erionite in the United States: An Emerging National Public Health Concern for Respiratory Disease." Environ. Geochem. Health 35 (4): 419-30.

[16] Townsend, D. W. 2008. "Combined Positron Emission Tomography-Computed Tomography: The Historical Perspective." Semin Ultrasound CT MR 29 (4): 232-5.

[17] Barreiro, T. J., and Katzman, P. J. 2006. "Malignant Mesothelioma: A Case Presentation and Review.” J. Am. Osteopath Assoc. 106 (12): 699-704.

[18] Restrepo, C. S., Vargas, D., Ocazionez, D., Martínez-Jiménez, S., Cuellar, S. L. B., and Gutierrez, F. R. 2013. "Primary Pericardial Tumors." Radiographics 33 (6): 1613-30.

[19] Roca, E., Laroumagnea, S., Vandemoortelea, T., Berdahb, S., Dutaua, H., Maldonadoe, F., et al. 2013. "18F-fluoro-2-deoxy-d-glucose Positron Emission Tomography/Computed Tomography Fused Imaging in Malignant Mesothelioma Patients: Looking from Outside Is not Enough.” Lung Cancer 79 (2): 187-90.

[20] Truong, M.T., Viswanathan, C., Godoy, M. B., Carter, B. W., and Marom, E. M. 2013. "Malignant Pleural Mesothelioma: Role of CT, MRI, and PET/CT in Staging Evaluation and Treatment Considerations." Semin Roentgenol. 48 (4): 323-34.

[21] Knuuttila, A., Kivisaari, L., Kivisaari, A., Palomäki, M., Tervahartiala, P., and Mattson, K. 2001. "Evaluation of Pleural Disease Using MR and CT. With Special Reference to Malignant Pleural Mesothelioma." Acta. Radiol. 42 (5): 502-7.

[22] Entwisle, J. 2004. "The Use of Magnetic Resonance Imaging in Malignant Mesothelioma." Lung Cancer 45: S69-71.

[23] Boraschi, P., Neri, S., Braccini, G., Gigoni, R., Leoncini, B., and Perri, G. 1999. "Magnetic Resonance Appearance of Asbestos-Related Benign and Malignant Pleural Diseases." Scand. J. Work Environ. Health 25 (1): 18-23.

[24] Ohno, Y. 2014. "New Applications of Magnetic Resonance Imaging for Thoracic Oncology." Semin. Respir. Crit. Care Med. 35 (1): 27-40.

[25] Van Zandwijk, N., Clarke, C., Henderson, D., Musk, A. W., Fong, K., Nowak, A., et al. 2013. "Guidelines for the Diagnosis and Treatment of Malignant Pleural Mesothelioma." J. Thorac. Dis. 5 (6): E254-307.

[26] Zahid, I., Sharif, S., Routledge, T., and Scarci, M. 2011. "What Is the Best Way to Diagnose and Stage Malignant Pleural Mesothelioma?.” Interact Cardiovasc Thorac Surg. 12 (2): 254-9.

[27] Wang, N., Zhai, H., and Lu, Y. 2013. "Is Fluorine-18 Fluorodeoxyglucose Positron Emission Tomography Useful for the Thyroid Nodules with Indeterminate Fine
Needle Aspiration Biopsy? A Meta-analysis of the Literature." J. Otolaryngol. Head \& Neck Surg. 42(1): 38.

[28] Orki, A., Akin, O., Tasci, A. E., Ciftci, H., Urek, S., Falay, O., et al. 2009. "The Role of Positron Emission Tomography/Computed Tomography in the Diagnosis of Pleural Diseases.” Thorac. Cardiovasc. Surg. 57 (4): 217-21.

[29] Kerger, B. D., James, R. C., and Galbraith, D. A. 2014. "Tumors that Mimic Asbestos-Related Mesothelioma: Time to Consider a Genetics-Based Tumor Registry?." Front Genet 5: 151.

[30] Patz, E. F. Jr., Shaffer, K., Piwnica-Worms, D. R., Jochelson, M., Sarin, M., Sugarbaker, D. J., et al. 1992. "Malignant Pleural Mesothelioma: Value of CT and MR Imaging in Predicting Resectability." AJR Am. J. Roentgenol. 159 (5): 961-6.

[31] Treglia, G., Sadeghi, R., Annunziata, S., Lococo, F., Cafarotti, S., Bertagna, F., et al., 2014. "Diagnostic Accuracy of 18F-FDG-PET and PET/CT in the Differential Diagnosis between Malignant and Benign Pleural Lesions: A Systematic Review and Meta-analysis." Acad. Radiol. 21 (1): 11-20.

[32] Plathow, C., Staab, A., Schmaehl, A., Aschoff, P., Zuna, I., Pfannenberg, C., et al. 2008. "Computed Tomography, Positron Emission Tomography, Positron Emission Tomography/Computed Tomography, and Magnetic Resonance Imaging for Staging of Limited Pleural Mesothelioma: Initial Results.” Invest. Radiol. 43 (10): 737-44.

[33] Ai, J., and Stevenson, J. P. 2014. "Current Issues in Malignant Pleural Mesothelioma Evaluation and Management." Oncologist 19 (9): 975-84.

[34] Feigen, M., Lee, S. T., Lawford, C., Churcher, K., Zupan, E., Scott, A. M., et al. 2011. "Establishing Locoregional Control of Malignant Pleural Mesothelioma Using High-Dose Radiotherapy and (18) F-FDG PET/CT Scan Correlation." J. Med. Imaging Radiat. Oncol. 55 (3): 320-32.

[35] Pehlivan, B., Topkan, E., Onal, C., Nursal, G. N., Yuksel, O., Dolek, Y., et al., 2009. "Comparison of CT and Integrated PET-CT Based Radiation Therapy Planning in Patients with Malignant Pleural Mesothelioma." Radiat. Oncol. 4: 35.

[36] Pilling, J., Dartnell, J. A., and Lang-Lazdunski, L. 2010. "Integrated Positron Emission Tomography-Computed Tomography Does not Accurately Stage Intrathoracic Disease of Patients Undergoing Trimodality Therapy for Malignant Pleural Mesothelioma." Thorac. Cardiovasc. Surg. 58 (4): 215-9.

[37] Otsuka, H., Terazawa, K., Morita, N., Otomi, Y., Yamashita, K., and Nishitani, H. 2009. "Is FDG-PET/CT Useful for Managing Malignant Pleural Mesothelioma?.” J. Med. Invest. 56 (1-2): 16-20. 
[38] Schaefer, N. G., Veit-Haibach, P., Soyka, J. D., Steinert, H. C., Stahel, R. A. 2012. "Continued Pemetrexed and Platin-Based Chemotherapy in Patients with Malignant Pleural Mesothelioma (MPM): Value of 18F-FDG-PET/CT.” Eur. J. Radiol. 81 (1): e19-25.

[39] Lee, H. Y., Hyun, S. H., Lee, K. S., Kim, B. T., Kim, J., Shim, Y. M., et al. 2010. "Volume-Based Parameter of 18)F-FDG PET/CT in Malignant Pleural Mesothelioma: Prediction of Therapeutic Response and Prognostic Implications.” Ann. Surg. Oncol. 17 (10): 2787-94.

[40] Genestreti, G., Moretti, A., Piciucchi, S., Giovannini, N., Galassi, R., Scarpi, E., et al., 2012. "FDG PET/CT Response Evaluation in Malignant Pleural Mesothelioma Patients Treated with Talc Pleurodesis and Chemotherapy." J. Cancer 3: 241-5.

[41] Veit-Haibach, P., Schaefer, N. G., Steinert, H. C., Soyka, J. D., Seifert, B., and Stahel, R. A. 2010. "Combined FDG-PET/CT in Response Evaluation of Malignant Pleural Mesothelioma." Lung Cancer 67 (3): 311-7.

[42] Niccoli-Asabella, A., Notaristefano, A., Rubini, D., Altini, C., Ferrari, C., Merenda, N., et al. 2013. "18F-FDG $\mathrm{PET} / \mathrm{CT}$ in Suspected Recurrences of Epithelial Malignant Pleural Mesothelioma in Asbestos-Fibers-Exposed Patients (Comparison to Standard Diagnostic Follow-Up)." Clin. Imaging 37 (6): 1098-103.

[43] Stewart, D. J., Martin-Ucar, A., Pilling, J. E., Edwards, J. G., O'Byrne, K. J., and Waller, D. A. 2004. "The Effect of Extent of Local Resection on Patterns of Disease Progression in Malignant Pleural Mesothelioma." Ann. Thorac. Surg. 78 (1): 245-52.

[44] Baldini, E. H., Recht, A., Strauss, G. M., DeCamp, M. M. Jr., Swanson, S. J., Liptay, M. J., et al. 1997. "Patterns of Failure after Trimodality Therapy for Malignant Pleural Mesothelioma." Ann. Thorac. Surg. 63 (2): 334-8.

[45] Tan, C., Barrington, S., Rankin, S., Landau, D., Pilling, J., Spicer, J., et al. 2010. "Role of Integrated 18-fluorodeoxyglucose Position Emission Tomography-Computed Tomography in Patients
Surveillance after Multimodality Therapy of Malignant Pleural Mesothelioma." J. Thorac. Oncol. 5 (3): 385-8.

[46] Bille, A., Chicklore, S., Okiror, L., Cook, G. J., Spicer, J., Landau, D., et al. 2013. "Patterns of Disease Progression on 18F-Fluorodeoxyglucose Positron Emission Tomography-Computed Tomography in Patients with Malignant Pleural Mesothelioma Undergoing Multimodality Therapy with Pleurectomy/Decortication." Nucl. Med. Commun. 34 (11): 1075-83.

[47] Lucignani, G., Paganelli, G., and Bombardieri, E. 2004. "The Use of Standardized Uptake Values for Assessing FDG Uptake with PET in Oncology: A Clinical Perspective." Nucl. Med. Commun. 25 (7): 651-6.

[48] Jadvar, H., and Parker, J. A. 2006. Clinical PET and PET/CT. Berlin: Springer.

[49] Masa-Ah, P., and Soongsathitanon, S. 2010. "A Novel Standardized Uptake Value (SUV) Calculation of PET DICOM Files Using MATLAB." In Proceedings of the 10th WSEAS International Conference on Applied Informatics and Communications and 3rd WSEAS International Conference on Biomedical Electronics and Biomedical Informatics 2010, 413-6.

[50] Flores, R. M., Akhurst, T., Gonen, M., Zakowski, M., Dycoco, J., Larson, S. M., et al. 2006. "Positron Emission Tomography Predicts Survival in Malignant Pleural Mesothelioma." J. Thorac. Cardiovasc. Surg. 132 (4): 763-8.

[51] Genestreti, G., Moretti, A., Piciucchi, S., Tiseo, M., Bersanelli, M., Scarlattei, M., et al. 2012. "Prognostic Value of 18F-FDG Standard Uptake Value by Integrated PET/CT in the Staging of Malignant Pleural Mesothelioma." Technol. Cancer Res. Treat. 11 (2): 163-7.

[52] Gerbaudo, V. H., Mamede, M., Trotman-Dickenson, B., Hatabu, H., and Sugarbaker, D. J. 2011. "FDG PET/CT Patterns of Treatment Failure of Malignant Pleural Mesothelioma: Relationship to Histologic Type, Treatment Algorithm, and Survival.” Eur. J. Nucl. Med. Mol. Imaging 38 (5): 810-21. 